\title{
Comparación entre los frotis de sangre central y sangre periférica para el diagnóstico de hemoparásitos en bovinos
}

\section{Comparison between the smears of central blood and peripheral blood for the diagnosis of hemoparasites in bovine}

\author{
Enrique Rimbaud Giambruno1, Maryuri Inés Mayorga-Escobar², Luis Miguel Guerrero Rojas ${ }^{3}$, Ana Cristina López \\ Badilla $^{3}$, Marcela Alejandra Vázquez Díaz ${ }^{3}$ \\ ${ }^{1}$ Prof. Dr. MVZ. Decano de la Facultad de Ciencias Agrarias. ORCID: https://orcid.org/0000-0003-3142-8862 \\ ${ }^{2}$ MSc. MVZ. Directora del laboratorio Centro de Diagnóstico e Investigación Veterinarias, Facultad de Ciencias Agrarias. ORCID: https:// \\ orcid.org/0000-0002-5350-1496 \\ ${ }^{3}$ Estudiante de Medicina Veterinaria y Zootecnia de la Facultad de Ciencias Agrarias. ORCDI: https://orcid.org/0000-0001-5986-0396 /OR- \\ CID: https://orcid.org/0000-0002-0485-0729 \\ Universidad de Ciencias Comerciales (UCC), Nicaragua
}

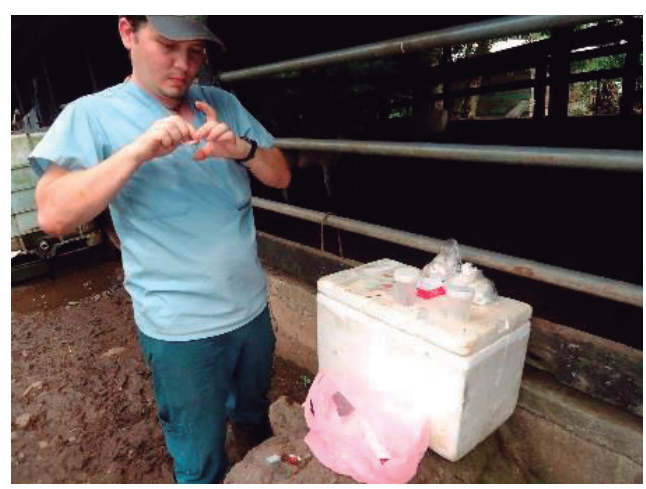

\section{RESUMEN}

Frente a desavenencias planteadas entre el diagnóstico clínico y laboratorial, se decide hacer un estudio comparativo entre el estudio diagnóstico de muestras de sangre central y periférica, para comparar resultados. Se muestrearon 100 vacas de diez fincas diferentes (10 vacas de cada finca), en la localidad de Pancasan, Matagalpa, Nicaragua, extrayendo de cada una, muestras de sangre central (vena yugular) y periférica (vena auricular). Todas las muestras fueron procesadas mediante frotis delgado, frotis de gota gruesa y visualización del capilar (Técnica de Woo), tiñéndolas tanto con los colorantes de Diff Quick, como con Giemsa. Los análisis de sangre central o periférica tienen errores muy grandes, mayores al $20 \%$, como para confiar plenamente en solo uno de ellos en el resultado de estudios sobre hemoparásitos. La sangre periférica solamente es más eficaz en el diagnóstico en un $2.79 \%$, lo que no es significativo $(p<0.1)$. Se recomienda realización de ambos análisis simultáneos (sangre central y periférica) para futuros diagnósticos de hemoparásitos en el laboratorio.

Palabras clave: diagnóstico, sangre, hemoparásitos, bovinos, sangre central, sangre periférica, Nicaragua

\section{ABSTRACT}

Faced with disagreements between the clinical and laboratory diagnosis, it was decided to make a comparative study between the diagnostic study of central and peripheral blood samples, to compare results. 100 cows were sampled from ten different farms (10 cows from each farm) in the town of Pancasan, Matagalpa, Nicaragua, extracting from each one samples of central blood (jugular vein) and peripheral blood (auricular vein). All the samples were processed by means of a thin smear, thick smear and capillary visualization (Woo technique), dyeing them both with the Diff Quick dyes and with Giemsa. The central or peripheral blood tests have very large errors, greater than $20 \%$, as to fully rely on only one of them in the results of studies on hemoparasites. Peripheral blood is only more effective in diagnosis at $2.79 \%$, which is not significant $(\mathrm{P}<0.1)$. It is recommended to carry out both simultaneous analyzes (central and peripheral blood) for future diagnoses of haemoparasites in the laboratory.

Key words: Diagnosis, blood, hemoparasites, bovines, central blood, peripheral blood, Nicaragua
Recibido: 16 de febrero del 2018

Aceptado: 25 de junio del 2018

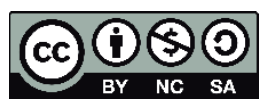

Los artículos de la revista La Calera de la Universidad Nacional Agraria, Nicaragua, se comparten bajo términos de la licencia Creative Commons: Reconocimiento, No Comercial, Compartir Igual. Las autorizaciones adicionales a las aquí delimitadas se pueden obtener en el correo freddy.aleman@ci.una.edu.ni

C Copyright 2018. Universidad Nacional Agraria 


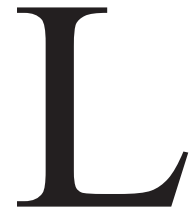

os hemoparásitos ocupan sin duda un lugar preponderante en la salud animal en Nicaragua, la prevalencia en todas las especies es bien alta y causa frecuentemente picos de mortandad (Rimbaud et al., 2017a; Rimbaud et al., 2017b).

El hemoparásito más frecuente en Nicaragua es Anaplasma sp., seguido de Babesia sp. y Tripanosoma sp. (Rimbaud et al., 2017a).

Internacionalmente, la bibliografía recomienda para el diagnóstico de laboratorio de hemoparásitos los frotis de sangre periférica, tanto sea en extendidos de frotis delgados como en gota gruesa (OMS, 1992; Richey y Palmer, 1990).

Frente a desavenencias planteadas entre el diagnóstico clínico y laboratorial, se decide hacer un estudio comparativo entre el estudio diagnóstico de muestras de sangre central y periférica, para comparar resultados.

\section{MATERIALES Y MÉTODOS}

Se muestrearon 100 vacas de diez fincas (10 vacas de cada finca), en la localidad de Pancasan, Matagalpa, Nicaragua, extrayendo de cada una, muestras de sangre central (vena yugular) y periférica (vena auricular).

Todas las muestras fueron procesadas mediante frotis delgado, frotis de gota gruesa y visualización del capilar (Técnica de Woo), tiñéndolas tanto con los colorantes de Diff Quick, como con Giemsa.

\section{RESULTADOS Y DISCUSIÓN}

En el cuadro 1 se observa claramente que ninguno de los dos métodos fue confiable en un $100 \%$. Se encontró un total de 72 animales positivos a Anaplasama sp, 3 positivos a Babesia sp., y 4 positivos a Tripanosoma sp.
Se consideraron solamente los resultados de Anaplasma sp. como significativos para el estudio.

Con el estudio de sangre central solo se diagnosticaron un $76.38 \%$ de los casos positivos, lo que indica un error de $23.62 \%$, inaceptable en diagnóstico laboratorial.

Con el estudio de sangre periférica solo se diagnosticaron un $79.16 \%$ de los casos positivos, lo que indica un error de $20.83 \%$, inaceptable en diagnóstico laboratorial.

La sangre periférica arrojó un \% de acierto de $2.79 \%$ superior que la sangre central, lo que no es significativo $(p<0.1)$.

La literatura internacional en su totalidad plantea como método diagnóstico de hemoparásitos los frotis de sangre periférica (Guillén et al., 2006).

Según Benavidez (2012), la probabilidad de detección de hemoparásitos se aumenta cuando se utilizan frotis de sangre capilar, pero este proceso debe realizarse en campo y requiere entrenamiento del personal.

La lectura de la gota gruesa o del extendido de sangre periférica tiene como objetivo establecer la presencia de hemoparásitos tales como Plasmodium, Babesia, Tripanosomas y algunas especies de Microfilarias. En el caso de determinar la presencia de Plasmodium, es de gran importancia determinar su especie y cuantificar el número de parásitos por $\mathrm{ml}$ de sangre, criterios que son básicos para el tratamiento y control del paciente (Las Américas, s.f.).

En un animal enfermo deben obtenerse muestras de sangre periférica para frotis, haciendo punción de la punta de la oreja o cola y de sangre con anticoagulantes para determinar hematocrito (SENASA, s.f.).

La sangre para el diagnóstico de hemoparásitos debe recogerse de una vena marginal y con el animal en fase febril (Gracia et al., 2006).

Cuadro 1. Comparación de resultados de análisis de sangre de origen central o periférico en bovinos y su contribución al diagnóstico de hemoparásitos

\begin{tabular}{|c|c|c|c|c|}
\hline & $\%$ Anaplasma & $\%$ Babesia & \% Tripanosoma & $\%$ Error \\
\hline Sangre Central & 55 & 3 & 2 & 17.00 \\
\hline $\begin{array}{l}\% \text { Diagnosticado del total de animales } \\
\text { afectados }\end{array}$ & 76.38 & 100 & 50 & 23.62 \\
\hline Sangre Periférica & 57 & 0 & 2 & 15.00 \\
\hline $\begin{array}{l}\% \text { Diagnosticado del total de animales } \\
\text { afectados }\end{array}$ & 79.16 & 0 & 50 & 20.83 \\
\hline Resultados compartidos & 41 & 0 & 2 & 31.00 \\
\hline Prevalencia Total & 72 & 3 & 4 & 2.79 \\
\hline
\end{tabular}


Estos resultados y recomendaciones no coinciden con nuestros resultados, encontrando en la investigación diferencias de resultados diagnóstico mayores al 20\% tanto en estudios de sangre central como periférica.

La sangre periférica tuvo una mejor performance de $2.79 \%$ frente a la sangre central, pero basados en el error evidenciado y el análisis estadístico vemos que el mismo no es significativo como para indicar que es mejor o más eficaz que el estudio de sangre central.

En base a los resultados, se recomendará la realización de ambos análisis (sangre central y periférica) para futuros diagnósticos de hemoparásitos en el laboratorio.

\section{CONCLUSIONES}

Los análisis de sangre central o periférica tienen errores muy grandes, mayores al $20 \%$, como para confiar plenamente en solo uno de ellos en el resultado de estudios sobre hemoparásitos.

La sangre periférica solamente es más eficaz en el diagnóstico en un $2.79 \%$, lo que no es significativo $(p<0.1)$.

Se recomienda realización de ambos análisis simultáneos (sangre central y periférica) para futuros diagnósticos de hemoparásitos en el laboratorio.

\section{REFERENCIAS BIBLIOGRÁFICAS}

Benavidez, E. 2012. Criterios y protocolos para el diagnóstico de hemoparásitos en bovinos. (en línea). Disponible en https://www. researchgate.net/publication/234047003_Criterios_y_protocolos_para_el_diagnostico_de_hemoparasitos_en_bovinos

Gracia, E; Chacón, G; Moreno, B; Fernández, A; Albizu, I; Baselga, R. 2006. Toma de muestras en rumiantes. Exopol. Autovacunas y Diagnóstico, San Mateo, Zaragoza. (en línea). Disponible en www.produccion-animal.com.ar

Guillén, A; León, E; Aragot, W; Silva, M. 2001. Diagnóstico de hemoparásitos en el instituto de investigaciones veterinarias periodo 1986-2000. Vet Trop 26(1):47-62.

Las Américas. (s.f.). Hematología general. (en línea). Disponible en http://www.lablasamericas.com.co/site/index.php/seccion/view/ hematologia general/examenes/H01/H350

OMS (Organización Mundial de la Salud). 1992. Métodos básicos de laboratorio en parasitología médica. 116 p.

Richey, EJ; Palmer, GH. 1990. Bovine anaplasmosis. Compendium on Continuing Education for the Practicing Veterinarian, 12(11):16611668.

Rimbaud, E; Mayorga, M, Sánchez, A, Picado, L. 2017a. Prevalencia de hemoparásitos en fincas de la zona de influencia de NICACENTRO. VIII congreso nacional ganadero, CONAGAN. Hotel Camino Real, 28 de julio de 2017.

Rimbaud, E; Sequeira, E; González, D; Mayorga, M. 2017b. Prevalencia de hematozoarios y parásitos gastrointestinales en animales de trabajo en Nicaragua. Boletín de Parasitología, 18(2):4

SENASA. s.f. Manual de Anaplasmosis y Babesiosis. (en línea). Disponible en http://www.senasa.gob.ar/manual-de-anaplasmosis-y-babesiosis. 